\title{
HISTORY OF STAR FORMATION OF EARLY TYPE GALAXIES FROM INTEGRATED LIGHT: STELLAR AGES AND ABUNDANCES
}

\author{
RicARDo P. Schiavon \\ Gemini Observatory, 670 N. A'ohoku Place, Hilo, HI 96720, USA \\ (Received July 21, 2010; Accepted August 3, 2010)
}

\begin{abstract}
I briefly review what has been learned from determinations of mean stellar ages and abundances from integrated light studies of early-type galaxies, and discuss some new questions posed by recent data. A short discussion of spectroscopic ages is presented, but the main focus of this review is on the abundances of $\mathrm{Fe}, \mathrm{Mg}, \mathrm{Ca}, \mathrm{N}$, and $\mathrm{C}$, obtained from comparisons of measurements taken in integrated spectra of galaxies with predictions from stellar population synthesis models.
\end{abstract}

key words: galaxies: elliptical and lenticulars; galaxies: stellar content; galaxies: abundances; galaxies: evolution

\section{INTRODUCTION}

How galaxies form - and the stars within them - is one of the major open questions of modern cosmology. Early-type galaxies (ETGs) host a large fraction of the stellar mass in today's universe, and typically show no evidence for major ongoing star formation, so they are natural targets for the investigation of how and when galaxy assembly and star formation occurred in the past. The task is a difficult one, which has consumed a large amount of observational and theoretical effort in the past several decades. All that work cannot be fairly summarized in a such short article. This review is thus narrowly focused on what has been learned from stellar ages and abundances based on integrated light studies, and the new questions raised by recent evidence. Apologies go to many hard-working colleagues for any important omissions.

\section{STELLAR AGES}

Historically, the debate on the star formation history of ETGs was framed in terms of two competing scenarios: hierarchical clustering (e.g., White \& Rees, 1978; Searle \& Zinn, 1978), according to which these galaxies were assembled through the merging of less massive structures formed at high redshift; and monolithic dissipative collapse (e.g., Eggen et al., 1962; Larson, 1974), whereby massive ETGs were formed at very high redshift by means of a rapid gravitational collapse. Deciding between these two scenarios was one of the main motivations behind attempts to measure stellar ages and abundances in ETGs. Today there is little question that galaxies formed hierarchically in a $\Lambda$-CDM universe, and while that historical debate has been settled, studies of unresolved stellar populations have acquired renewed importance, as they provide much needed constraints to increasingly sophisticated galaxy formation models.

Early attempts at dating stellar populations from applications of stellar population synthesis models to observations of integrated light were based on photometric or low-resolution spectrophotometric observations (e.g., O’Connell, 1980; Gunn et al., 1981; Renzini \& Buzzoni, 1986) and high-resolution photographic spectroscopy (e.g., Rose, 1985). They led to promising, yet not entirely conclusive results, due to limitations of the early models and/or uncertainties associated with the age-metallicity degeneracy (e.g., Renzini \& Buzzoni, 1986; Worthey, 1994). The latter is a manifestation of the similar dependence of the temperatures of main sequence and giant stars of a given stellar population on age and metallicity, which causes the integrated 
colors of stellar populations, particularly in the optical and near-UV, to respond in similar ways to variations of these two parameters.

It was only after the systematic modeling of Balmer lines as (relatively) clean age indicators, initiated by Worthey (1994), that reliable quantitative estimates of mean luminosity-weighted ${ }^{1}$ stellar ages became available. The method relies on the dependence of Balmer lines, such as $\mathrm{H} \beta \lambda 486 \mathrm{~nm}$, on the temperatures and luminosities of turnoff stars - which are higher in younger stellar populations. For A-type stars and cooler, Balmer line strength is positively correlated with temperature, so that the lines in integrated spectra of stellar populations older than a few 100 Myr are stronger for younger ages. Spectroscopic ages based on $H \beta$ for large numbers of ETGs, both in the field and in clusters, suggest that many of them have undergone recent star formation events (e.g., Trager et al., 2000; Kuntschner, 2000; Caldwell et al., 2003; Denicoló et al., 2005; Thomas et al., 2005). Trager et al. proposed a scenario where the bulk of the stars in their sample galaxies were old, and only a very small fraction of their stellar populations had young ages ( $\sim 1$ Gyr $)$. Because the latter are brighter in the optical, they weight the mean ages towards lower values. Although plausible, this schematic scenario could not be verified by the Trager et al. data because of the degeneracy between the age of the young component and its relative contribution to a galaxy's total stellar mass budget, which cannot be broken on the basis of $H \beta$ and metal-line indices alone.

With the inclusion of additional age indicators, such as higher-order Balmer lines in the blue (Leonardi \& Rose, 1994; Worthey \& Ottaviani, 1997; Schiavon, 2007), stronger constraints were placed on the age distributions of stars. These studies confirmed early suggestions that a small fraction of the stellar mass budget (few \%) is required to be young in order to match the data. They also ruled out claims that Balmer line strengths in the spectra of ETGs required the presence of metal-poor stars with blue horizontal branches (e.g., Freitas Pacheco \& Barbuy, 1995; Maraston \& Thomas, 2000; Lee et al., 2000). Models including metal-poor stars cannot simultaneously match all

\footnotetext{
${ }^{1}$ See Trager \& Somerville (2009) for a discussion of the relation between of luminosity- and mass-weighted ages/abundances with those obtained from comparison of line indices with single stellar population synthesis models.
}

Balmer and metal lines in the 400-530 $\mathrm{nm}$ range (see also Trager et al., 2005).

Perhaps the cleanest evidence for the presence of young/intermediate-age stellar populations was provided by Galex observations of ETGs by Yi et al. (2005). Analyzing UV-optical colors of ETGs from the Sloan Digital Sky Survey, Yi et al. found that approximately $1 / 3$ of their sample present strong UV fluxes which cannot be explained by hot horizontal branch stars. They estimate that approximately $1-2 \%$ of the stellar mass is in the form of young stars. Because they constitute a tiny fraction of the stellar mass budget, young stars are vastly outshone by older populations in the optical, where they can only be detected on the basis of accurate spectrum synthesis of very high S/N spectra (e.g., Schiavon et al., 2004; Schiavon, 2007; Graves et al., 2007) — while being relatively easy to detect in the UV.

More recent estimates based on a combination of Galex photometry with larger SDSS samples indicate that as much as $10 \%$ of all stellar mass in ETGs today were formed since $\mathrm{z} \sim 1$ ( $\mathrm{S}$. Yi, private communication). While a fascinating debate is ongoing regarding what triggered and quenched this relatively recent star formation (e.g., Kuntschner et al., 2010; Zhu et al., 2010; Sánchez-Blázquez et al., 2009; Serra et al., 2008; Schawinski et al., 2007; Kaviraj et al., 2007; Graves et al., 2007), information on the history of formation of the remaining $\sim 90 \%$ of the stellar mass is relatively scanty, possibly because it requires observations of large samples at higher redshifts than so far possible and/or a far more thorough assessment of the fossil record in the abundance patterns of stars in the nearby ETGs. The latter is the topic of the next section.

\section{STELLAR ABUNDANCES}

Crucial information about the star formation history is encoded in the chemical composition of stars. The key observables are mean (luminosity-weighted) metallicities, abundance ratios, and the run of these quantities with $\sigma$. It has long been known that the central regions of nearby massive galaxies are metal-rich (e.g., Spinrad \& Taylor, 1971). It was only far more recently that reliable metallicity estimates became possible for massive ETGs at cosmological distances, showing that they have comparably high metallicities, (e.g., Spinrad et al., 1997; Jørgensen et al., 2005; Schiavon et al., 2006), implying a relatively rapid early chemical en- 
richment. A more detailed knowledge of the history of star formation requires accurate estimates of detailed abundance patterns in a range of redshifts, but work on abundance ratios has so far been restricted to relatively nearby samples (but see Jørgensen et al., 2005 and Kelson et al., 2006).

\subsection{Magnesium}

The Mg I 517-518 nm doublet has been the chief metallicity indicator in early studies of the chemical composition of ETGs from integrated light, due to its strength and location in a spectral region where astronomical detectors were very sensitive. Early studies found evidence for an overabundance of $\mathrm{Mg}$ relative to $\mathrm{Fe}$ in ETGs (e.g., Peterson 1976). When $\mathrm{Mg}$ and Fe lines were finally compared with models of stellar population synthesis, it was found that $[\mathrm{Mg} / \mathrm{Fe}]^{2}$ is above solar in the centers of massive ETGs (Worthey et al., 1992). This was arguably one of the most influential results in the history of the field, and it has guided theoretical work to this day. Further studies showed that Mg enhancement is correlated with central velocity dispersion $(\sigma)$ and metallicity (e.g., Trager et al., 2000; Thomas et al., 2005; Schiavon, 2007; Smith et al., 2009). At least three scenarios have been invoked to explain this finding, all involving the balance between $\mathrm{Mg}$ enrichment by SN II and Fe enrichment by SN Ia: short star formation timescale, top-heavy IMF, and selective winds (Faber et al., 1992). One popular interpretation of the data invokes the existence of a relation between star formation timescale and galaxy mass (e.g., Thomas et al., 2005). The data, however, do not rule out scenarios based on selective winds or IMF. A definitive answer still awaits better data and more realistic chemodynamical models (see further discussion in Section 3.3).

\subsection{Calcium}

Past studies suggest that $\mathrm{Ca}$ does not behave like $\mathrm{Mg}$, with $[\mathrm{Ca} / \mathrm{Fe}]$ being possibly solar (or lower) and not correlated with $\sigma$. Trager et al. (1998) found the Lick/IDS Ca4227 index to be essentially independent of $\sigma$. Accordingly, Thomas et al. (2003) concluded

\footnotetext{
${ }^{2}$ More often than not, this result is phrased in the literature in terms of an overabundance of $\alpha$ elements relative to iron. Despite the many theoretical reasons in favor of the assumption that all $\alpha$ elements should vary in tandem, there is so far no evidence that any $\alpha$ element other than $\mathrm{Mg}$ is enhanced in ETGs, except perhaps for Ti (Milone et al., 2000).
}

that $[\mathrm{Ca} / \mathrm{Fe}]$ in their sample galaxies was also essentially constant with $\sigma$. Saglia et al. (2002), on the other hand, found the Ca II triplet (CaT, 849, 855, $862 \mathrm{~nm})$ to be mildly decreasing with $\sigma$. Vazdekis et al. (2003) and Cenarro et al. (2004) compared new single stellar population synthesis models for the CaT with data for field and Coma galaxies, again finding very low $[\mathrm{Ca} / \mathrm{Fe}]$. While difficult to understand, given that both $\mathrm{Ca}$ and $\mathrm{Mg}$ are $\alpha$ elements manufactured in similar (though not identical) nucleosynthetic sites, the implications of these results are potentially important, giving theorists ample room for a wide range of speculations.

The unexpected behavior of Ca seems to be instead most likely caused by difficulties in the interpretation of the measurements, particularly because the two $\mathrm{Ca}$ indices employed in these studies do not respond to $\mathrm{Ca}$ abundance variations in a clean fashion. Prochaska et al. (2005) showed that the Ca4227 index is severely affected by a CN bandhead which contaminates the blue pseudocontinuum of the index, making it lower. Because $\mathrm{CN}$ is strongly correlated with $\sigma$ (Trager et al., 1998), the effect is stronger for higher $\sigma$ galaxies, offsetting any dependence of the Ca line strength itself on $\sigma$, thus making the index $\sigma$-independent. Prochaska et al. demonstrated this by defining a new index, Ca4227 $r$, which is less affected by $\mathrm{CN}$ contamination. They showed that Ca4227 $r$ is as strongly correlated with $\sigma$ as $\mathrm{Mg} b$.

While the issue of the slope of the Ca4227- $\sigma$ relation is seemingly resolved, models that account for the effect of $\mathrm{CN}$ on the $\mathrm{Ca} 4227$ index still indicate $[\mathrm{Ca} / \mathrm{Fe}] \sim 0$ in massive ETGs (Schiavon, 2007; Graves et al., 2007). At face value, this confirms the abundance ratios found by previous studies. However, there may be non-negligible systematics in the Ca abundances derived by application of the Schiavon (2007) models. They are affected by uncertainties in age, and in the abundances of Fe, $\mathrm{C}$, and N. They are also affected by uncertainties in the way models account for the contamination of Ca4227 by $\mathrm{CN}$. So the matter should be considered far from settled.

Regarding the results based on CaT, one should bear in mind that the integrated spectra of metal-rich stellar populations in the CaT region is dominated by $\mathrm{M}$ giants (Schiavon \& Barbuy, 1999), and that fact has implications for both the zero point and the slope of the $[\mathrm{Ca} / \mathrm{Fe}]-\sigma$ relation. First let us consider the zero point. 
The stellar libraries employed in the models used to analyze CaT data in the past contain hardly any $\mathrm{M}$ giants with known metallicity, let alone [Ca/Fe] (Cenarro et al., 2001a). Therefore, $[\mathrm{Ca} / \mathrm{Fe}]$ in the models themselves is uncertain, which obviously makes it very hard for one to infer reliable $[\mathrm{Ca} / \mathrm{Fe}]$ from comparison of those models with the data. As regards the slope of the $\mathrm{CaT}-\sigma$ relation, we recall that the CaT lines are located in a region where opacity in the spectra of $\mathrm{M}$ giants is dominated by $\mathrm{TiO}$ lines. While the definition of the $\mathrm{CaT}^{\star}$ index employed in these studies is partly meant to account for $\mathrm{TiO}$ contamination (Cenarro et al., 2001b), the index has not been shown to be immune to variations in $[\mathrm{Ti} / \mathrm{Fe}]^{3}$ which may be important, given that there is evidence that $\mathrm{Ti}$ is enhanced in ETGs (Milone et al., 2000). Regarding the negative slope of the $\mathrm{CaT}^{\star} \sim \sigma$ relation, that could be due to the effect of $\mathrm{TiO}$ opacity on the pseudocontinuum, because: (1) $\mathrm{TiO}$ is well correlated with $\sigma$ (Trager et al., 1998), and (2) TiO lines are more sensitive to metallicity than CaT lines (Schiavon et al., 2000; Schiavon \& Barbuy, 1999; Jørgensen et al., 1992). Finally, CN contamination of the CaT indices may also be important (Erdelyi-Mendes \& Barbuy, 1991).

In summary, we suggest that $\mathrm{Ca}$ abundances are far from well known in ETGs, and there is no compelling motivation to resort to extreme scenarios to account for the numbers currently available in the literature. More work is needed to produce reliable $[\mathrm{Ca} / \mathrm{Fe}]$ in ETGs.

\subsection{Nitrogen \& Carbon}

While the behavior of $\mathrm{C}$ - and $\mathrm{N}$-sensitive indices such as Lick $\mathrm{CN}_{1}, \mathrm{CN}_{2}, \mathrm{G} 4300$ and $\mathrm{C}_{2} 4668$ in ETG spectra has been well documented for over a decade, it was only after the Schiavon (2007) models and their implementation in EZ_Ages (Graves \& Schiavon, 2008) that these indices could be interpreted in terms of $[\mathrm{N} / \mathrm{Fe}]$ and $[\mathrm{C} / \mathrm{Fe}]$ (see also Kelson et al., 2006). Both abundance ratios are found to be super-solar and correlate strongly with $\sigma$ and metallicity (Schiavon, 2007; Graves et al., 2007; Smith et al., 2009). This result has been called into question recently by Toloba et al. (2009), who found no correlation between the strength of the near-UV NH3360 feature and $\sigma$ in a sample of nearby galaxies. They argue that the NH3360 band is a clearer indicator of $\mathrm{N}$ abundance than the Lick CN features

\footnotetext{
${ }^{3} \mathrm{TiO}$ lines are very sensitive to variations in the abundance of Ti.
}

used by EZ_Ages, because the latter are also dependent on $\mathrm{C}$ abundance. The absence of a slope in the NH3360 - $\sigma$ relation may be explained by the presence of metalpoor stars, whose contribution to the integrated light is highest in the UV. In fact, multiple stellar population models show that the inclusion of a small fraction of a metal-poor population flattens the NH3360 $-\sigma$ relation, even in the presence of a $[\mathrm{N} / \mathrm{Fe}]-\sigma$ correlation (G. Worthey, 2010, private communication).

The existence of a steep slope in the $[\mathrm{N} / \mathrm{Fe}]-\sigma$ relation, if confirmed, is an important result, as it may indicate secondary enrichment of $\mathrm{N}$ by stars ranging from $4-8 \mathrm{M}_{\odot}$ (Chiappini et al., 2003). Because these stars last for $\sim 10^{8}$ years, the presence of a secondaryenrichment signature in the chemical composition of stars in ETGs may constrain the lower limit for the duration of star formation in the systems that formed the stars that live today in those galaxies, (Schiavon, 2007) and, perhaps most importantly, their characteristic masses. The increasing evidence for the presence of multiple stellar populations in globular clusters (Piotto, 2009) may be an important clue in this regard. It has been long known that there is a marked spread in $\mathrm{N}$ and $\mathrm{C}$ abundances in globular cluster stars (e.g., Smith \& Norris, 1982; Cannon et al., 1998; Carretta et al., 2005), which is roughly consistent with enrichment by intermediate mass stars going through the AGB phase (Ventura \& D'Antona, 2008). The presence of such CN inhomogeneities seems to be a function of both cluster mass and environment (Martell \& Smith, 2009), as predicted by recent models (Conroy \& Spergel, 2010).

One may reasonably speculate that the evidence above indicates that the stars we see in nearby ETGs were formed in the precursors of today's Galactic globular clusters. In that scenario, the signature of secondary $\mathrm{N}$ enrichment we see in ETGs today would have been established in the precursors of globular clusters, before they merged to form the massive, dynamically hot systems we see today. The Galactic halo can be used as a resolved proxy to test this scenario. The recent identification of CN bimodality in a sample of halo field stars by Martell \& Grebel (2010) argues for a similar process in operation during the formation of the Galactic halo. Because CN - strong stars have almost certainly been formed in globular clusters (or there precursors in the distant past), their presence in the halo field is evidence of the early dissolution of those systems in the formation of the Galactic halo. Martell \& Grebel estimate 
that as much as $50 \%$ of the halo mass may have been contributed by globular clusters and their precursors. If a similar process was responsible for the assembly of stellar mass in ETGs, one might wonder whether a similar fraction of the total mass would have been contributed by the globular cluster precursors. Could the slope of the $[\mathrm{N} / \mathrm{Fe}]$ vs. $[\mathrm{Fe} / \mathrm{H}]$ be used to constrain that number? What were the characteristic masses of those systems? Would it be possible to construct chemodynamical evolution models for those low-mass systems that are capable of reproducing all the abundance ratios measured in today's ETGs?

Inclusion of $\mathrm{C}$ and $\mathrm{N}$ in abundance analyses of ETGs is bringing interesting new insights on their star formation histories, which could potentially even lead to a reinterpretation of the data on $[\mathrm{Mg} / \mathrm{Fe}]$. Smith et al. (2009) analyzed a large data set for galaxies from the Coma cluster and Shapley supercluster, spanning a very wide range of $\sigma$. They determined the abundances of several elements using EZ_Ages, then performed biparametric fits to the relation between $[\mathrm{Mg}$, $\mathrm{Ca}, \mathrm{C}, \mathrm{N} / \mathrm{Fe}]$ and both $[\mathrm{Fe} / \mathrm{H}]$ and $\sigma$. Smith et al. found that both $[\mathrm{Mg} / \mathrm{Fe}]$ and $[\mathrm{Ca} / \mathrm{Fe}]$ decrease with $[\mathrm{Fe} / \mathrm{H}]$, whereas $[\mathrm{N} / \mathrm{Fe}]$ and $[\mathrm{C} / \mathrm{Fe}]$ do not correlate with it. They suggest that the run of $[\mathrm{Mg} / \mathrm{Fe}]$ and $[\mathrm{Ca} / \mathrm{Fe}]$ with $[\mathrm{Fe} / \mathrm{H}]$ indicates a short time scale for star formation. They consider that the lack of correlations of $[\mathrm{N} / \mathrm{Fe}]$ and $[\mathrm{C} / \mathrm{Fe}]$ with $[\mathrm{Fe} / \mathrm{H}]$ is expected since, unlike $\mathrm{Mg}$ and $\mathrm{Ca}, \mathrm{C}$ and $\mathrm{N}$ are contributed by low(er) mass stars, so that these elements should scale with $\mathrm{Fe}$, not with $\mathrm{Mg}$ and $\mathrm{Ca}$. Interestingly, on the other hand, all abundance ratios show a strong correlation with $\sigma$. Smith et al. contend that this result is difficult to interpret in terms of a simple dependence of star formation timescale on galaxy mass (e.g., Thomas et al., 2005), because that would preclude a correlation between $[\mathrm{C} / \mathrm{Fe}]$ and $[\mathrm{N} / \mathrm{Fe}]$ with $\sigma$. Clearly, more work is needed to clarify this issue.

\section{CONCLUDING REMARKS}

The discussion above highlights the power of stellar population synthesis to infer mean stellar properties from integrated light, and constrain galaxy formation models. Presently, well tested stellar population synthesis models can be used to determine mean stellar ages and abundances of $\mathrm{Fe}, \mathrm{Mg}, \mathrm{Ca}, \mathrm{C}$, and $\mathrm{N}$. The recent addition of the latter two elements may spark the emergence of a more complex picture of galaxy forma- tion. In order for that to happen, more work is needed to refine chemodynamical models used to interpret the abundance measurements and their relation with global galaxy properties in terms of the physics of galaxy formation. There has been very promising recent progress on this front, which, due to space limitations, could not be reviewed here (e.g., Arrigoni et al., 2010; Pipino et al., 2009a, b). More theoretical work is also needed to develop better stellar population synthesis models to ascertain the reality of current abundance determinations, and to include more elements in the pool of reliable abundances. On this front as well, different groups are making steady progress (Lee et al., 2009; Coelho et al., 2007; Peterson, 2007). In that regard, it is particularly desirable to extend stellar population synthesis modelling towards the UV, in order to match the upcoming observing capabilities that will make possible collection of large samples of galaxy spectra at redshift beyond 2. With the expected developments in theory and observations, this field will likely go through very exciting times in the next decade.

\section{ACKNOWLEDGEMENTS}

The author thanks Yonsei University and the organizers, especially Suk-Jin Yoon and Sukyoung Yi, for a truly delightful workshop. The hospitality of the Department of Astrophysical Sciences at Princeton University, where this paper was partly conceived, is warmly acknowledged. Conversations with David Spergel, Charlie Conroy, Jenny Graves, and Richard McDermid contributed substantially to the formulation of some of the ideas presented in this paper.

\section{REFERENCES}

Arrigoni, M., Trager, S. C., Somerville, R. S., \& Gibson, B. K., 2010, Galactic Chemical Evolution in Hierarchical Formation Models - I. Early-Type Galaxies in the Local Universe, MNRAS, 402, 173

Caldwell, N., Rose, J. A., \& Concannon, K. D., 2003, Star Formation Histories of Early-Type Galaxies. I. Higher Order Balmer Lines as Age Indicators, AJ, 125, 2891

Cannon, R. D., et al., 1998, Carbon and Nitrogen Abundance Variations on the Main Sequence of 47 Tucanae, MNRAS, 298, 601

Carretta, E., et al., 2005, Abundances of C, N, O in Slightly Evolved Stars in the Globular Clusters NGC 6397, NGC 6752 and 47 Tuc, A\&A, 433, 597 
Cenarro, A. J., Cardiel, N., Gorgas, J., Peletier, R. F., Vazdekis, A., \& Prada, F., 2001b, Empirical Calibration of the Near-Infrared Ca II Triplet I. The Stellar Library and Index Definition, MNRAS, 326, 959

Cenarro, A. J., Gorgas, J., Cardiel, N., Pedraz, S., Peletier, R. F., \& Vazdekis, A., 2001a, Empirical Calibration of the Near-Infrared Ca II Triplet - II. The Stellar Atmospheric Parameters, MNRAS, 326,981

Cenarro, A. J., Sánchez-Blázquez, P., Cardiel, N., \& Gorgas, J., 2004, Early-Type Galaxies in the Coma Cluster: A New Piece in the Calcium Puzzle, ApJ, 614, L101

Chiappini, C., Romano, D., \& Matteucci, F., 2003, Oxygen, Carbon and Nitrogen Evolution in Galaxies, MNRAS, 339, 63

Coelho, P., Bruzual, G., Charlot, S., Weiss, A., Barbuy, B., \& Ferguson, J. W., 2007, Spectral Models for Solar - Scaled and $\alpha$ - Enhanced Stellar Populations, MNRAS, 382, 498

Conroy, C. \& Spergel, D. N., 2010, On the Formation of Multiple Stellar Populations in Globular Clusters, ApJ, submitted, arXiv:1005.4934

Denicoló, G., Terlevich, R., Terlevich, E., Forbes, D. A., \& Terlevich, A., 2005, Group, Field and Isolated Early-Type Galaxies - II. Global Trends from Nuclear Data, MNRAS, 358, 813

Eggen, O. J., Lynden-Bell, D., \& Sandage, A. R., 1962, Evidence from the Motions of Old Stars that the Galaxy Collapsed, ApJ, 136, 748

Erdelyi-Mendes, M. \& Barbuy, B., 1991, Synthetic Ca II Infrared Triplet Lines as a Function of Stellar Parameters, A\&A, 241, 176

Faber, S. M., Worthey, G., \& González, J. J., 1992, Absorption-Line Spectra of Elliptical Galaxies and Their Relation to Elliptical Formation, Proc. IAU Symp. 149, (Dordrecht:Kluwer) p. 255

Freitas Pacheco, J. A. \& Barbuy, B., 1995, Horizontal Branch Morphology and $\mathrm{H}_{\beta}$ Indices of Globular Clusters, A\&A, 302, 718

Graves, G. J., Faber, S. M., Schiavon, R. P., \& Yan, R., 2007, Ages and Abundances of Red Sequence Galaxies as a Function of LINER Emission-Line Strength, ApJ, 671, 243

Graves, G. J. \& Schiavon, R. P., 2008, Measuring Ages and Elemental Abundances from Unresolved Stellar Populations: $\mathrm{Fe}, \mathrm{Mg}, \mathrm{C}, \mathrm{N}$, and $\mathrm{Ca}, \mathrm{ApJS}$, 177,446
Gunn, J. E., Stryker, L. L., \& Tinsley, B. M., 1981, Evolutionary Synthesis of the Stellar Population in Elliptical Galaxies. III - Detailed Optical Spectra, ApJ, 249, 48

Jørgensen, I., et al., 2005, RX J0152.7-1357: Stellar Populations in an X-Ray Luminous Galaxy Cluster at $\mathrm{z}=0.83$, AJ, 129, 1249

Jørgensen, U. G., Carlsson, M., \& Johnson, H. R., 1992, The Calcium Infrared Triplet Lines in Stellar Spectra, A\&A, 254, 258

Kaviraj, S., et al., 2007, UV-Optical Colors as Probes of Early-Type Galaxy Evolution, ApJS, 173, 619

Kelson, D., Illingworth, G. D., Franx, M., \& van Dokkum, P. G., 2006, Line Strengths in EarlyType Cluster Galaxies at $\mathrm{z}=0.33$ : Implications for $\alpha / \mathrm{Fe}$, Nitrogen, and the Histories of E/S0s, ApJ, 653, 159

Kuntschner, H., 2000, The Stellar Populations of Early-Type Galaxies in the Fornax Cluster, MNRAS, 315, 184

Kuntschner, H., et al., 2010, The SAURON Project - XVII. Stellar Population Analysis of the Absorption Line Strength Maps of 48 Early-Type Galaxies, MNRAS, in press, arXiv:1006.1574

Larson, R. B., 1974, Dynamical Models for the Formation and Evolution of Spherical Galaxies, MNRAS, 166, 585

Lee, H. -C., Yoon, S. -J., \& Lee, Y. -W., 2000, The H $\beta$ Index as an Age Indicator of Old Stellar Systems: The Effects of Horizontal-Branch Stars, AJ, 120, 998

Lee, H. -C., et al., 2009, Stellar Population Models and Individual Element Abundances. II. Stellar Spectra and Integrated Light Models, ApJ, 694, 902

Leonardi, A. J. \& Rose, J. A., 1996, The Ages of Starbursts in Post-Starburst Galaxies, AJ, 111, 182

Maraston, C. \& Thomas, D., 2000, Strong Balmer Lines in Old Stellar Populations: No Need for Young Ages in Ellipticals?, ApJ, 541, 126

Martell, S. L. \& Smith, G. H., 2009, CN Variations in High-Metallicity Globular and Open Clusters, PASP, 121, 577

Martell, S. L. \& Grebel, E. K., 2010, Light-Element Abundance Variations in the Milky Way Halo, A\&A, in press, arXiv:1005.4070

Milone, A., Barbuy, B., \& Schiavon, R. P., 2000, The Ratio of $\alpha$-Elements to Iron in Early-Type Galaxies from $\mathrm{TiO}$ and $\mathrm{Mg}_{2}$, AJ, 120, 131

O'Connell, R. W., 1980, Galaxy Spectral Synthesis. II - M32 and the Ages of Galaxies, ApJ, 236, 430 
Peterson, R. C., 1976, Overabundances of Sodium and the Heavy Isotopes of Magnesium in StrongLined Elliptical Galaxies, ApJ, 210, L123

Peterson, R. C., 2007, Constraining Star Formation in Old Stellar Populations from Theoretical Spectra, ASP Conference Series, 374, 369

Piotto, G., 2009, Observations of Multiple Populations in Star Clusters, Proc. IAU Symp., 258, 233

Pipino, A., Chiappini, C., Graves, G., \& Matteucci, F., 2009a, Stellar Mass Loss, Rotation and the Chemical Enrichment of Early-Type Galaxies, MNRAS, 396, 1151

Pipino, A., Devriendt, J. E. G., Thomas, D., Silk, J., \& Kaviraj, S., 2009b, GALICS. II: the $[\alpha / \mathrm{Fe}]-$ Mass Relation in Elliptical Galaxies, A\&A, 505, 1075

Prochaska, L. C., Rose, J. A., \& Schiavon, R. P., 2005, A New Definition for the Ca4227 Feature: Is Calcium Really Underabundant in Early-Type Galaxies?, AJ, 130, 2666

Renzini, A. \& Buzzoni, A. 1986, in Spectral Evolution of Galaxies, C. Chiosi \& A. Renzini, Eds. (Dordrecht:Reidel), 151

Renzini, A. \& Buzzoni, A. 1986, Global Properties of Stellar Populations and the Spectral Evolution of Galaxies, Ibid., ASSL, 122, 195

Rose, J. A., 1985, Constraints on Stellar Populations in Elliptical Galaxies, AJ, 90, 1927

Rose, J. A., 1994, The Integrated Spectra of M32 and of 47 Tuc: A Comparative Study at High Spectral Resolution, AJ, 107, 206

Saglia, R. P., Maraston, C., Thomas, D., Bender, R., \& Colless, M., 2002, The Puzzlingly Small Ca II Triplet Absorption in Elliptical Galaxies, ApJ, 579, L13

Sánchez-Blázquez, P., Gibson, B. K., Kawata, D., Cardiel, N., \& Balcells, M., 2009, Are Dry Mergers Dry, Moist or Wet?, MNRAS, 400, 1264

Schawinski, K., et al., 2007, Observational Evidence for AGN Feedback in Early-Type Galaxies, MNRAS, 382, 1415

Schiavon, R. P., 2007, Population Synthesis in the Blue. IV. Accurate Model Predictions for Lick Indices and UBV Colors in Single Stellar Populations, ApJS, 171, 146

Schiavon, R. P. \& Barbuy, B., 1999, The Temperature Scale of Metal-Rich M Giants Based on TiO Bands: Population Synthesis in the NearInfrared, ApJ, 510, 934

Schiavon, R. P., Barbuy, B., \& Bruzual, A. G., 2000 Near-Infrared Spectral Features in Single-Aged Stellar Populations, ApJ, 532, 453

Schiavon, R. P., Caldwell, N., \& Rose, J. A., 2004, The Integrated Spectrum of M67 and the Spectroscopic Age of M32, AJ, 127, 1513

Schiavon, R. P., et al., 2006, The DEEP2 Galaxy Redshift Survey: Mean Ages and Metallicities of Red Field Galaxies at z 0.9 from Stacked Keck DEIMOS Spectra, ApJ, 651, L93

Searle, L. \& Zinn, R., 1978, Compositions of Halo Clusters and the Formation of the Galactic Halo, ApJ, 225, 357

Serra, P., Trager, S. C., Oosterloo, T. A., \& Morganti, R., 2008, Stellar Populations, Neutral Hydrogen, and Ionised Gas in Field Early-Type Galaxies, A\&A, 483, 57

Smith, G. H. \& Norris, J., 1982, The Cyanogen Distributions in NGC 3201, M55, and M71, ApJ, 254, 149

Smith, R. J., Lucey, J. R., Hudson, M. J., \& Bridges, T. J., 2009, Abundance Ratios in Red-Sequence Galaxies over a Wide Mass Range: the 'X-Planes' for Magnesium, Calcium, Carbon and Nitrogen, MNRAS, 398, 119

Spinrad, H. \& Taylor, B. J., 1971, The Stellar Content of the Nuclei of Nearby Galaxies. I. M31, M32, and M81, ApJS, 22, 445

Spinrad, H., et al., 1997, LBDS 53W091: an Old, Red Galaxy at $\mathrm{z}=1.552, \mathrm{ApJ}, 484,581$

Thomas, D., Maraston, C., \& Bender, R., 2003, New Clues on the Calcium Underabundance in EarlyType Galaxies, MNRAS, 343, 279

Thomas, D., Maraston, C., Bender, R., \& Mendes de Oliveira, C., 2005, The Epochs of Early-Type Galaxy Formation as a Function of Environment, ApJ, 621, 673

Toloba, E., Sánchez-Blázquez, P., Gorgas, J., \& Gibson, B. K., 2009, Carbon and Nitrogen Abundances in Early-Type Galaxies, ApJ, 691, 95

Trager, S. C., Worthey, G., Faber, S. M., Burstein, D., \& González, J. J., 1998, Old Stellar Populations. VI. Absorption-Line Spectra of Galaxy Nuclei and Globular Clusters, ApJS, 116, 1

Trager, S. C., Faber, S. M., Worthey, G., \& González, J. J., 2000, The Stellar Population Histories of Local Early-Type Galaxies. I. Population Parameters, AJ, 119, 1645

Trager, S. C., Worthey, G., Faber, S. M., \& Dressler, A., 2005, Hot Stars in Old Stellar Populations: A Continuing Need for Intermediate Ages, MNRAS, 362,2 
Trager, S. C. \& Somerville, R. S., 2009, Probing Recent Star Formation with Absorption-Line Strengths in Hierarchical Models and Observations, MNRAS, 395, 608

Vazdekis, A., Cenarro, A. J., Gorgas, J., Cardiel, N., \& Peletier, R. F., 2003, Empirical Calibration of the Near-Infrared CaII Triplet - IV. The Stellar Population Synthesis Models, MNRAS, 340, 1317

Ventura, P. \& D'Antona, F., 2008, Self-Enrichment by Asymptotic Giant Branch Stars in Globular Clusters: Comparison between Intermediate and High Metallicities, MNRAS, 385, 2034

White, S. D. M. \& Rees, M. J., 1978, Core Condensation in Heavy Halos - A Two-Stage Theory for Galaxy Formation and Clustering, MNRAS, 183, 341

Worthey, G., 1994, Comprehensive Stellar Population Models and the Disentanglement of Age and Metallicity Effects, ApJS, 95, 107

Worthey, G., Faber, S. M., \& González, J. J., 1992, $\mathrm{Mg}$ and $\mathrm{Fe}$ Absorption Features in Elliptical Galaxies, ApJ, 398, 69

Worthey, G. \& Ottaviani, D. L., 1997, $\mathrm{H} \gamma$ and $\mathrm{H} \delta$ Absorption Features in Stars and Stellar Populations, ApJS, 111, 337

Zhu, G., Blanton, M. R., \& Moustakas, J., 2010, Stellar Populations of Elliptical Galaxies in the Local Universe, ApJ, submitted, arXiv:1006.5055

Yi, S. K., et al., 2005, Galaxy Evolution Explorer Ultraviolet Color-Magnitude Relations and Evidence of Recent Star Formation in Early-Type Galaxies, ApJ, 619, L111 